\title{
Appetite and physiological responses to medium-chain triglycerides (MCT) and conjugated linoleic acid (CLA) in lean vs overweight/obese participants
}

\author{
$\underline{\text { Tyler Maher }}^{1}$, Martina Deleuse ${ }^{1}$, Sangeetha Thondre $^{1}$ and Miriam Clegg ${ }^{1,2}$ \\ ${ }^{1}$ Oxford Brookes University, Oxford, United Kingdom and \\ ${ }^{2}$ University of Reading, Reading, United Kingdom
}

\section{Abstract}

Medium-chain triglycerides (MCT) are lipids which have been shown to yield appetite-suppressing properties compared to long-chain triglycerides (LCT), which are more commonly found in the diet. Conjugated linoleic acid (CLA) has also been shown to increase satiety and decrease subsequent energy intake, although CLA has received much less attention to date. The mechanisms behind the appetite-suppressing properties are yet to be fully elucidated. Furthermore, no study has directly compared these effects or mechanisms in overweight/obese individuals to a group of healthy-weight weight controls.

Fifteen healthy-weight (BMI: $22.7 \pm 1.9 \mathrm{~kg} / \mathrm{m}^{2}$ ) and fourteen overweight/obese (BMI: $30.9 \pm 3.9 \mathrm{~kg} / \mathrm{m}^{2}$ ) individuals participated in a randomised single-blind cross-over study. Overnight fasted participants were fed smoothie breakfasts containing either $23.06 \mathrm{~g}$ vegetable oil (LCT), $25.0 \mathrm{~g}$ MCT oil (MCT), or $6.25 \mathrm{~g} \mathrm{CLA}$ and $16.8 \mathrm{~g}$ vegetable oil (CLA) on separate test days. Subjective appetite ratings, peptide YY (PYY), total ghrelin, $\beta$-hydroxybutyrate, and gastric emptying (GE) were measured throughout each trial. Energy intake in the laboratory was measured at a buffet meal 3-h after the smoothie breakfast and for the following 36-h in their free-living environment via food records.

Energy intake and subjective appetite ratings were not affected by weight status, so results are presented as a whole. MCT reduced energy intake compared to LCT over the trial day and whole 48-h period, but not at the ad libitum lunch. There was no difference between CLA and LCT at any time point. There were no differences in postprandial subjective appetite ratings between trials. PYY concentrations were not affected by weight status $(P=0.170)$, and did not differ between trials $(P=0.743)$ and total ghrelin was also not affected by weight status $(P=0.306)$, and concentrations were similar between trials $(P=0.188)$. $\beta$-hydroxybutyrate concentrations were higher in the overweight/obese group compared to the lean group $(P=0.009)$, and MCT led to increased concentrations compared to both LCT $(P=0.005)$ and CLA $(P<0.001)$. MCT delayed GE half-time $(P<0.001)$, lag-phase $(P<0.001)$, latency phase $(P=0.01)$, and ascension time $(P<0.001)$ compared to LCT, with no differences in GE between LCT and CLA (all $P>0.05)$.

MCT consumption reduces intake over the subsequent 48-h, whereas CLA has no effect compared to LCT. This does not appear to be related to PYY or total ghrelin, but may be mediated by $\beta$-hydroxybutyrate or via a delay of gastric emptying.

\section{Conflict of Interest}

There is no conflict of interest. 